\title{
EEGG: An analytic brain-computer interface algorithm
}

\author{
Gang Liu, Jing Wang \\ Institute of Robotics and Intelligent Systems, Xi'an Jiaotong University, Xi'an, China \\ E-mail: Gangliu.6677@gmail.com; wangpele@gmail.com
}

\begin{abstract}
Objective. In the traditional sense, the modeling approaches can be divided into white-box (physics-based), black-box (data-driven), and gray-box (the combination of physics-based and data-driven). Because the human brain is a black box itself, the EEG-BCI algorithm is generally a data-driven approach. It generates a black-box or gray-box (e.g., "Visualizing convolutional networks") model. However, one black- or gray-box cannot completely explain the brain. This paper presents the first analytic "white-box" EEG-BCI algorithm using Gang neurons (EEGG).

Approach. Independent and interactive components of neurons or brain regions can fully describe the brain. This paper constructed a relationship frame based on the independent and interactive compositions for intention recognition and analysis using a novel dendrite module of Gang neuron. A total of 4,906 EEG data about motor imagery (MI) of left-hand and righthand movements from 26 subjects were obtained from GigaDB. Firstly, this paper explored EEGG's classification performance according to cross-subject accuracy. Secondly, this paper transformed the EEGG model into a relation spectrum expressing independent and interactive components of brain regions. Then, the relation spectrum was verified through the known ERD/ERS phenomenon. Finally, this paper explored the previously unreachable further BCIbased analysis of brain.

Main results. (1) EEGG was more robust than typical "CSP+" algorithms for the data of poor quality [AUC:0.825 $\pm 0.074(\mathrm{EEGG})>0.745 \pm 0.094(\mathrm{CSP}+\mathrm{LDA}) / 0.591 \pm 0.104(\mathrm{CSP}$

+Bayes $/ 0.750 \pm 0.091(\mathrm{CSP}+\mathrm{SVM}), p<0.001]$. (2) The relation spectrum showed the

known ERD/ERS phenomenon. (3) Interestingly, EEGG showed that brain regions' interactive components put a brake on ERD/ERS effects for classification $(p<0.001)$. This means that generating fine hand intention needs more centralized activation in the brain.

Significance. EEGG decomposed the biological EEG-intention system of this paper into the relation spectrum inheriting Taylor series, rather than the fuzzy interpretation of outputs, which offers a novel frame for analysis of the brain.
\end{abstract}

Keywords: Brain-computer interface, Gang neuron, white box, EEG, analysis

\section{Introduction}

Brain-computer interface (BCI) based on electroencephalogram (EEG) is a technology translating EEG signals that record user's brain activity into control commands [1-3]. Central to the theory of BCI is the model of the biological EEG-intention system. In the traditional sense, the modeling approaches can be divided into white-box (physics-based), black-box (data-driven), and gray-box (the combination of physics-based and data-driven) [4, 5]. Biologists and clinicians sometimes more welcome the white-box models because they are easily understood and can be used to analyze biological mechanisms [6]. However, most of the existing EEG-based BCIs are data-driven because specific physical properties of intention are still a mystery. Restricted by basic machine learning (ML) 
algorithms, such as Support Vector Machines (SVM) [7-9], Neural Network (NN), and Bayesian Classifier [10], traditional EEG-based BCIs usually model the biological EEG-intention system as a black-box $[8,11]$.

The studies of interpretability of ML outputs, such as feature visualization for deep networks [12, 13], attribution for convolutional networks [14, 15], and dimensionality reduction [16], are emerging in computer vision and natural language processing in recent years. Moreover, interpretable approaches have been used to explain the BCI model's outputs [17-20]. For instance, Layerwise relevance propagation (LRP) was used to explain individual deep network decisions/outputs in the literature [17]. However, it is necessary here to clarify exactly that the "Interpretation" refers to roughly explain the outputs of the black-box model (e.g., deep neural networks) [17] rather than read the parameters of the model. Therefore, it only conforms to the definition of gray-box [5].

One of the most significant challenges of science today is to unlock the brain's mysteries (i.e., the biological EEGintention system) [21]. Nevertheless, the black- or gray-box model cannot resolve the brain's specific properties, and the modeling of the traditional white-box model requires known and specific brain properties. Therefore, this is an endless loop.

\section{- Introduction of Scheme}

Fortunately, decomposing an unknown composition into some simple components with physical meaning (e.g., Fourier transform in signal processing) has already proved to be effective in tackling this kind of unknown and complicated problems [22]. In addition to the trigonometric series (Fourier transform), Taylor series also have physical meaning. For example, items of Taylor series contain $x_{1}$ (first-order independent component), $x_{1}^{2}$ (second-order independent component), and $x_{1} x_{2}$ (second-order interactive component), etc ., where $x_{1}$ and $x_{2}$ are independent variables. In 2020, Gang Liu et al. proposed a basic machine learning algorithm, Dendrite Net (DD) [23]. The central concept of Dendrite Net is that the algorithm can recognize this class after learning, if the output's logical expression contains the corresponding class's logical relationship among inputs (andlorlnot) [23]. Interestingly, the model using Dendrite Net can be translated into Relation Spectrum consisting of independent components and interactive components (Taylor series).

Independent and interactive components of neurons or brain regions can fully describe the brain. Therefore, the independent and interactive components are regarded as simple components with a physical meaning. This paper proposed a white-box relation frame based on brain regions' independent and interactive components using a dendrite module of Gang neuron (an improved Dendrite Net). EEGG decomposed the biological EEG-intention system [24]. Therefore, it can be used for intention recognition and analysis of the biological EEG-intention system. This paper addresses the following major questions of this algorithm/frame.

(1) This paper compared the classification performance of EEGG with other classic algorithms and further explored its advantages, disadvantages, and theoretical reasons for the performance.

(2) Since this paper decomposed the EEG intention system into a relation spectrum (Taylor-like series) for the first time, the rationality of EEGG decomposition was verified by exploring whether the components of the decomposed EEG-intention system contained the known ERD/ERS phenomenon.

(3) Based on the rationality, this paper explored the previously unreachable further BCI-based analysis of brain.

This paper is organized as follows. Section 2 introduces EEGG and experiments. Section 3 presents the results. Section 4 discusses the results. Conclusions and outlook are given in Section 5.

\section{Methods}

This section is divided into two sub-sections: EEGG frame and Experiments. EEGG frame section consists of Model building for classification and Transformation of model for generating relation spectrum. Experiments section consists of Experimental architecture and Experimental details.

\subsection{EEGG frame}

\subsubsection{Model building for classification}

The architecture of the EEGG Model for classification is shown in Fig. 1. It should be noted that the "Spatial filter" and "Power calculation" of EEGG refer to the implementation of the corresponding functions of previous single algorithms rather than using the corresponding single algorithms (e.g., CSP) [25]. EEGG Model is an end-to-end model. The input of EEGG is the EEG signal after band-pass filtering, and there is no need for separate spatial filtering, feature extraction, or other processing. The independent and interactive components of brain regions are stored in weights $W$. Weights are optimized by minimizing loss function using backpropagation (Recommended optimizer: SGD in PyTorch). The mean-square error (MSE) was the loss function used in this paper. In the rest of this section, we will introduce each step in detail. 


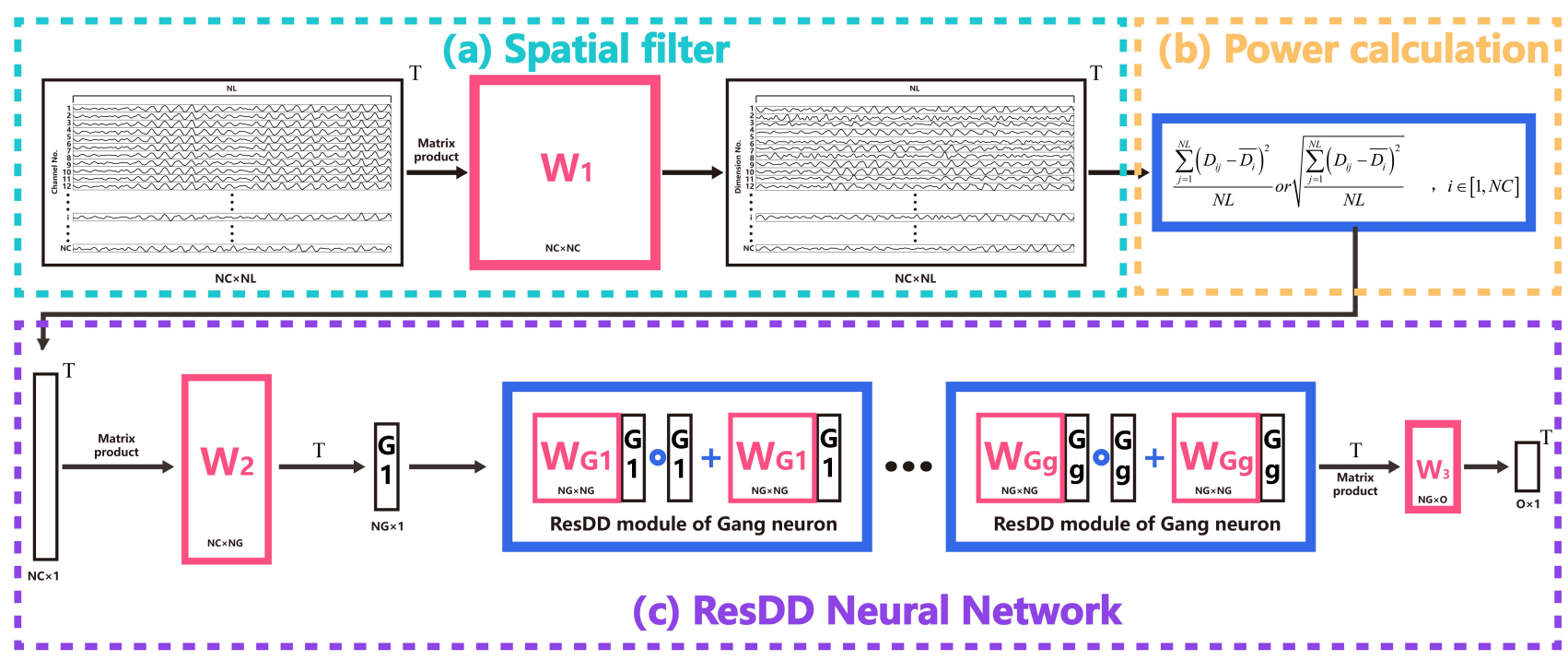

Figure 1. EEGG model building for classification. (a) Spatial filter. (b) Power calculation. (c) ResDD Neural Network. It should be noted that the "Spatial filter" and "Power calculation" of EEGG refer to the implementation of the corresponding functions of previous single algorithms rather than using the corresponding single algorithms (e.g., CSP). The input of EEGG is the EEG signal after band-pass filtering, and there is no need for separate spatial filtering, feature extraction, or other processing. $N C$ : the number of channels. $N L:$ the number of data points for each channel. $N G$ : the dimensions of inputs of ResDD. $g$ : the number of ResDD modules. $O$ : the dimensions of outputs. Note that EEGG Model (the above figure) is an end-to-end model, and the input of the EEGG model is the EEG data of a time window to be detected or classified.

\section{(a) Spatial filter (see Fig.1a)}

Raw EEG scalp potentials are known to have a poor spatial resolution due to volume conduction [26]. Therefore, spatial filters are extremely useful in the BCI model, especially motor imagination-based BCI, in order to improve the signal-to-noise ratio [25]. Spatial filters aim to project the signal $E^{\text {Input }}(t) \in \mathbb{R}^{N C}$ in the original sensor space to $E^{\text {Spatial fitering }}(t) \in \mathbb{R}^{N C}$, which lives in the surrogate sensor space, as follows:

$$
E^{\text {Spatial filtering }}(t)=\left(E^{\text {Input }}(t)\right)^{\mathrm{T}} W_{1}
$$

Where $W_{1} \in \mathbb{R}^{N C \times N C}$ is the weight matrix of the spatial filter. $N C$ is the number of channel. A traditional spatial filter is a single algorithm (e.g., CSP, PCA, and ICA) [25, 27]. The weight matrix $W_{1}$ of traditional spatial filter can be solved in two ways: first, an EEG-data-driven and unsupervised manner (e.g., PCA and ICA) [27], and second, a simple data-driven and supervised manner (e.g., CSP) [25]. However, traditional solution methods were not associated with later feature extraction and classification algorithms, making the weight matrix $W_{1}$ not optimal for later processing steps. Therefore, in the EEGG frame, the spatial filter solution is integrated into the end-to-end model. The weight matrix of the spatial filter is associated with power calculation and the ResDD neural network.

\section{(b) Power calculation (see Fig.1b)}

After spatial filtering, we calculate the power of each channel of $E^{\text {Spatial filtering }}(t)$. Unlike the previous method, the unsigned characteristic of EEG signals in the record is taken into account. EEGG uses the variance or standard error instead of the magnitude squared to remove the direct current (DC) component [28]. Likewise, power calculation is also integrated into the end-to-end model rather than a single algorithm. The formulas are as follows.

$$
P_{i}=\frac{\sum_{j=1}^{N L}\left(D_{i j}-\overline{D_{i}}\right)^{2}}{N L} \operatorname{sr} \sqrt{\frac{\sum_{j=1}^{N L}\left(D_{i j}-\overline{D_{i}}\right)^{2}}{N L}} \quad, i \in[1, N C]
$$

Where $P_{i} \in \mathbb{R}$ is the power of the $i-$ th channel, $D_{i j} \in \mathbb{R}^{N C \times N L}$ expresses the $j$-th data point of the $i-$ th channel. $N C$ is the number of channels. After the power calculation step, a $1 \times N C$ vector is obtained.

\section{(c) ResDD Neural Network (see Fig.1c)}

A traditional artificial neuron was expressed as $f(w x+b)$ or $f(W X)$ where $w, b, x, W$, and $X$ expressed the weight, bias, inputs, weight matrix, and inputs with element 1 , respectively, and $f$ was activation function. This artificial neuron was designed by the stereotypical 


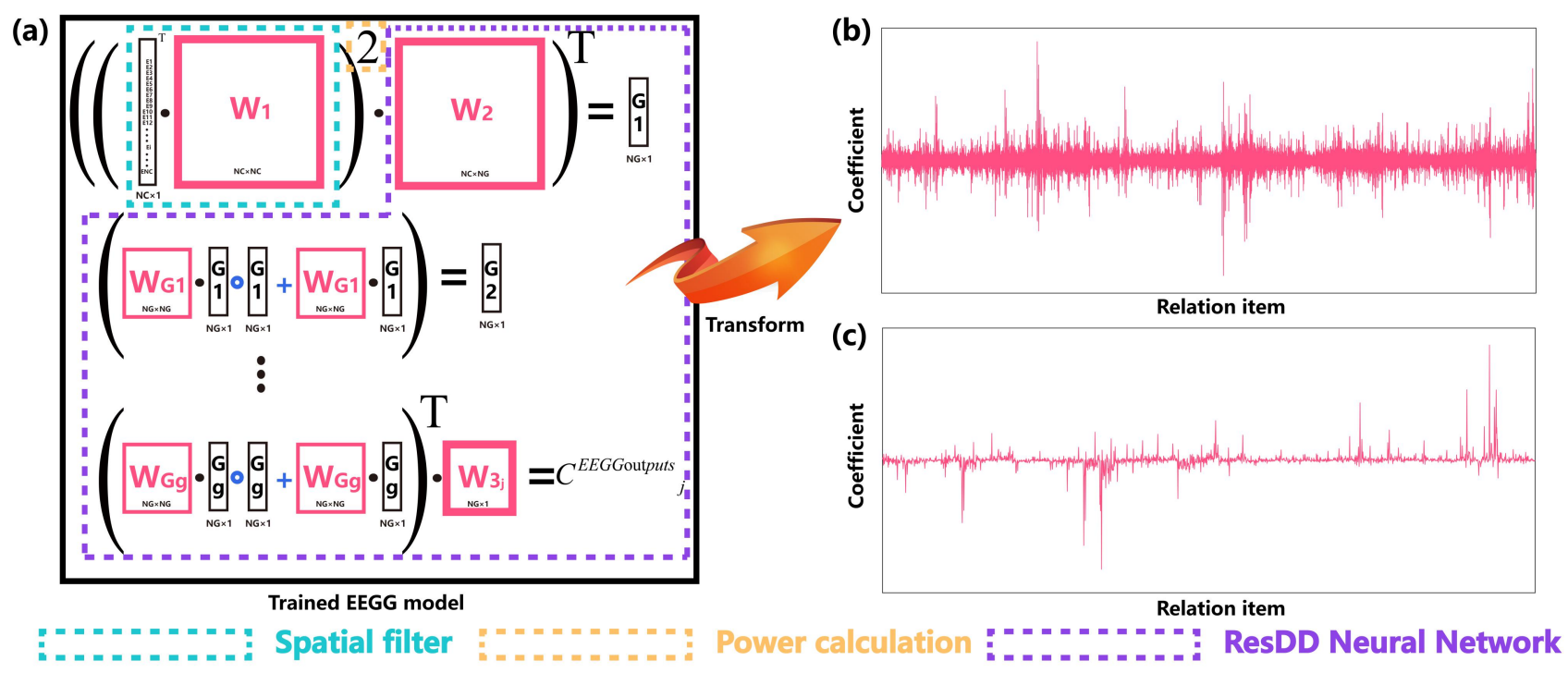

Figure 2. Transformation of model for generating relation spectrum. (a) The transformation of EEGG model. The step aims to transform trained EEGG model into simple components with physical meaning. (b) The relation spectrum form of EEGG model. By analogy to Fourier spectrum [abscissa: frequency $F$ (i.e., $\sin (2 \pi F t)$ or $\cos (2 \pi F t)$ ).ordinate: amplitude (i.e.,coefficient).], the abscissa of relation

spectrum is items of independent components and interactive components [e.g., $E_{1}$ (first-order independent component), $E_{1}^{2}$ (secondorder independent component), $E_{1} E_{2}$ (second-order interactive component), etc ., where $E_{1}$ and $E_{2}$ are EEG signals.]. (c) The relation spectrum removing the odd items. Odd items: e.g., $E_{1}, E_{1}^{2} E_{2}, E_{1} E_{2}^{2}$. Even items: e.g., $E_{1}{ }^{2}, E_{1}{ }^{2} E_{2}{ }^{2} . C_{i}$ :the $i-$ th channel. $N C$ : the number of channels. $N G$ : the dimensions of inputs of ResDD. $g:$ the number of ResDD modules. $C_{j}^{E E G G o u t p u t s}:$ the $j-$ th output.

knowledge of biological neurons 70 years ago and did not consider dendrites' information processing capacity. However, in 2020, Albert Gidon et al. demonstrated that biological dendrites participate in the pre-calculation of input data [29]. Subsequently, a Gang neuron was presented [24]. ResDD is one of the dendrite modules in Gang neurons. Previously, Dendrite Net was proposed, and its nonlinear mapping, generalization capability, and identification capability have been proved by experiment and theory (open source code) [23]. ResDD is a improved Dendrite Net using a residual strategy to prevent a vanishing gradient. [24, 30]. Therefore, ResDD inherits controllable nonlinear mapping and can be simplified after weight optimization because there is no nonlinear function in it. The work presented in this paper is the first application of ResDD module of Gang neuron.

ResDD Neural Network in this paper contains a dimensionality reduction module, ResDD modules, and one linear module whose dimensions are determined by the number of categories. The role of the dimensionality reduction module is to project power $P_{i} \in \mathbb{R}^{N C}$ onto $N G$ dimensions to reduce computational complexity ( $N G<N C$ ). The formula of the dimensionality reduction module is as follows.

$$
P^{G 1}=\left(P W_{2}\right)^{\mathrm{T}}
$$

Where $P^{G 1} \in \mathbb{R}^{N G \times 1} \quad$ expresses the outputs of the dimensionality reduction module. $W_{2} \in \mathbb{R}^{N C \times N G}$ is the weight matrix.

ResDD modules aim for nonlinear mapping. The number of ResDD modules $g$ can adjust the degree of interaction terms. The $i-$ th ResDD module is expressed as follows.

$$
P^{G i+1}=W_{G i} P^{G i} \circ P^{G i}+W_{G i} P^{G i}, i \in[1, g], i \in \mathbb{N}^{+}
$$

Where $P^{G i} \in \mathbb{R}^{N G \times 1}, P^{G i+1} \in \mathbb{R}^{N G \times 1}$ expresses the inputs and outputs of the $i$-th ResDD module, respectively. $W_{G i} \in \mathbb{R}^{N G \times N G}$ is the weight matrix. 。 expresses Hadamard product.

The last linear module is used to achieve the mapping of category. The formula is as follows.

$$
C^{E E G \text { Goutputs }}=\left(P^{G g+1}\right)^{T} W_{3}
$$

Where $P^{G g+1} \in \mathbb{R}^{N G \times 1}$ is the outputs of the last ResDD module. $C^{E E G G \text { outputs }} \in \mathbb{R}^{1 \times O}$ is the outputs of EEGG. The number of categories determines dimensions $O . W_{3} \in \mathbb{R}^{N G \times O}$ is the weight matrix.

It is important to stress once more that EEGG Model is an end-to-end model, the input of the EEGG model is the EEG data of a time window to be detected or classified, and 
Weights of EEGG are optimized by minimizing loss function using backpropagation (see Fig.1).

\subsubsection{Transformation of model for generating relation spectrum}

This part aims to transform the trained EEGG model into simple components with physical meaning, which is similar to the purpose of Fourier transform (see Fig. 2). However, Fourier transform was used for signal decomposition, while EEGG was used to decompose the EEG-intention system.

Since the DC component has been removed in building the EEGG model, the EEG variables of the EEGG model are regarded as the ideal state without the DC component. The power of EEG is calculated by the magnitude squared instead of the variance or standard error. Therefore, neither Spatial filter, Power calculation, nor ResDD Neural Network contains a nonlinear function. Nevertheless, interestingly, EEGG implements nonlinear operations by the Matrix product and Hadamard product. In order to decompose the EEG-intention system, the input variables $E=\left[E_{1}, E_{2}, \cdots, E_{N C}\right]$ and the output variable $C^{E E G G \text { outputs }}{ }_{j}$ of system are set. Then, the trained EEGG model is represented by the formulae containing simple arithmetic operations according to the model building steps(see "2.1.1 Model building for classification" and Fig. 2).

$$
\left\{\begin{array}{c}
P^{G 1}=\left(\left(E W_{1}\right)^{2} W_{2}\right)^{T} \\
P^{G 2}=W_{G 1} P^{G 1} \circ P^{G 1}+W_{G 1} P^{G i} \\
\vdots \\
P^{G i+1}=W_{G i} P^{G i} \circ P^{G i}+W_{G i} P^{G i} \\
\vdots \\
C^{E E G G \text { outputs }}=\left(W_{G g} P^{G g} \circ P^{G g}+W_{G g} P^{G g}\right)^{T} W_{3_{j}}
\end{array}\right.
$$

Where $W_{1} \in \mathbb{R}^{N C \times N C}$ is the weight matrix of the spatial filter. $W_{2} \in \mathbb{R}^{N C \times N G}$ is the weight matrix of the dimensionality reduction module. $W_{G 1} \in \mathbb{R}^{N G \times N G}, \ldots, W_{G i} \in \mathbb{R}^{N G \times N G}, \ldots$, $W_{G g} \in \mathbb{R}^{N G \times N G}$ are the weight matrixes of ResDD modules. $\left[\begin{array}{lllll}W_{3_{1}} & \cdots & W_{3_{j}} & \cdots & W_{3_{10}}\end{array}\right]=W_{3} \in \mathbb{R}^{N G \times O}$ is the weight matrix of the last linear module for categories. $W_{3_{j}} \in \mathbb{R}^{N G}$ denotes the weight vector of the $j$-th output. $P^{G 1}, \ldots, P^{G i}, \ldots$, $P^{G g}$ are intermediate variables. $g$ denotes the number of ResDD modules. $\circ$ expresses Hadamard product.

The weight matrixes in Eq.(6) have been optimized by minimizing loss function using backpropagation during training the EEGG model. Therefore, the optimized weights are assigned to the corresponding matrixes or vectors in Eq.(6). Then the relation spectrum was obtained through formula simplification of Eq.(6) in software (e.g., MATLAB or Python).

Fig. $2 b$ shows the relation spectrum form of the EEGG model. By analogy to Fourier spectrum [abscissa: frequency $F$ (i.e., $\sin (2 \pi F t)$ or $\cos (2 \pi F t)$ ).ordinate: amplitude (i.e., coefficient).], the abscissa of relation spectrum is items of independent components and interactive components [e.g., $E_{1}$ (first-order independent component), $E_{1}^{2}$ (second-order independent component), $E_{1} E_{2}$ (secondorder interactive component), etc ., where $E_{1}$ and $E_{2}$ are EEG signals.]. Because of the unsigned characteristic of EEG signals in the record, odd items' effects are random. Thus, odd items are removed, and the new relation spectrum is shown in Fig.2c [Odd items: e.g., $E_{1}, E_{1}^{2} E_{2}, E_{1} E_{2}^{2}$. Even items: e.g., $E_{1}^{2}, E_{1}^{2} E_{2}^{2}$.].

\subsection{Experiments}

\subsubsection{Experimental architecture}

The experiments will be undertaken to answer the following research question.

(a) This paper compared the classification performance of EEGG with other classic algorithms and further explored its advantages, disadvantages, and theoretical reasons for the performance.

Firstly, this paper performed the statistical test on all subjects' classification performance together (see Fig. 3). Secondly, in order to explore the advantages of EEGG deeper (to explore EEGG's advantages, disadvantages, and reasons), this paper divided the subjects into EEGG advantaged and disadvantaged groups (group (a) and group (b)). This paper explored the algorithm differences within the group to determine whether the grouping is reasonable (see Fig. 4 and Fig. 5). Thirdly, this paper compared the differences between the EEGG advantaged and disadvantaged groups (see Fig. 6).

(b) Since this paper decomposed the EEG intention system into a relation spectrum (Taylor-like series) for the first time, the rationality of EEGG decomposition was verified by exploring whether the components of the decomposed EEG-intention system contained the known ERD/ERS phenomenon.

Firstly, EEGG models were transformed into the new relation spectrum form. Then, this paper checked the number of relation items to verify if the actual number matches with the theoretical number. Secondly, in order to verify the universality and rationality of EEGG models, this paper computed the correlation matrix between the subjects' EEGG models (see Fig.7, Comparation of EEGG models). Last but 
most importantly, in order to screen statistically significant items, one-sample t-tests under $p<0.01$ and $p<0.001$ were applied to compare each item's mean to zero value (see Fig.8, statistical test of items across EEGG models, MATLAB code: ttest), because randomized effect approaches zero. Fig. 8 showed the items that differed significantly from zero. Further, this paper visualized the statistically significant items using the BrainNet Viewer $(p<0.001)$ (see Fig.9) [31].

The rationality of EEGG decomposition was verified by exploring whether the statistically significant items contained the known ERD/ERS phenomenon.

(c) Based on rationality, this paper explored the previously unreachable further BCI-based analysis of brain.

This paper analyzed the EEG- intention system based on the statistically significant items of the relation spectrum.

\subsubsection{Experimental details}

This section consists of Subjects, Data preprocessing, EEGG parameter and contrast models, and Classification performance. Among them, model parameter and contrast models are all described in Data processing.

\section{(a) Subjects}

It is also well known that there is event-related desynchronization/synchronization (ERD/ERS) in the motor imagery paradigm [32-34]. Therefore, this paper used ERD/ERS in the MI paradigm to confirm EEGG's analysis capacity. Additionally, there are many open MI datasets [32]. However, based on our knowledge of the MI paradigm, not all MI datasets notice essential details, such as BCIinefficient subjects [35], inefficient MI [36], and mode of imagery [37]. Therefore, this paper selected the dataset from GigaDB [32] as we consider that this dataset took into account the above necessary details [32, 38]. This dataset contains EEG data for MI of the left and right hands with 52 subjects. As shown in Fig. 9, a 64-channel montage based on the international 10-10 system was used to record the EEG signals with $512 \mathrm{~Hz}$ sampling rates [32]. Data with the noise electrodes and inefficient MI trials were marked. The most important detail was that subjects of this dataset were explicitly asked to imagine the kinesthetic experience rather than imagining the visual experience [32, 37]. Besides, an algorithm with high accuracy for a subject could perform terribly for other subjects, which is called a cross-subject problem [39]. Thus, this paper tested cross-subject accuracy. $1 / 2$ of the dataset (26 subjects) were randomly selected, and each subject was tested in turns. The testing subject's data were testing data, and other subjects' data were used to train models.

\section{(b) Data preprocessing}

Firstly, this paper removed the trial data with noise and intercepted the data from $0.5 \mathrm{~s}$ after the MI cue to $3 \mathrm{~s}$. Secondly, the obtained data were filtered with a band-pass filter from 8 to $30 \mathrm{~Hz}$. Finally, the obtained data were normalized between -1 and 1 in each trial. Besides, this problem was a two-class problem essentially, and thus this paper labeled the right-hand class as -1 and labeled the lefthand class as +1 [3]. The above procedure was repeated for data of all subjects. At this point, this paper obtained the data structure of every single trial of all subjects: EEG data $E \in \mathbb{R}^{64 \times 1281}$, label $C \in \mathbb{R}$.

\section{(c) EEGG parameter and contrast models}

According to the data structure of the single trial, the hyperparameters of EEGG were as follows: the number of channels $N C$ was set as 64 , the number of data points for each channel $N L$ was set as 1281 , the dimensions of inputs of ResDD $N G$ was set as 2, the number of ResDD modules $g$ was set as 1 , the dimensions of outputs $O$ was set as 1 .

This paper did not emphasize that the classification accuracy of EEGG was better than SOAT and mainly emphasized EEGG's decomposition capability. Moreover, a systematic review of the literature showed that deep learning methods have not yet demonstrated convincing improvement [3]. Therefore, this paper selected three typical "CSP+" BCI algorithms as contrast models: CSP and LDA (CSP+LDA), CSP and Naive Bayes classifier (CSP+Bayes), and CSP and SVM (CSP+SVM). Contrast models was actualized via CSP Toolbox and Statistics and Machine Learning Toolbox in MATLAB R2019a (CSP code: csp, LDA code: fitcdiscr, Bayes code: fitcnb, SVM code: fitcsvm) [40]. Among them, the SVM used a radial basis function Kernel (RBF).

\section{(d) Classification Performance}

The receiver operating characteristic (ROC) curve and the area under the curve (AUC) were used for performance measures [3]. Besides, because this study aimed to compare the differences between EEGG and other algorithms, and the data for comparing EEGG with other algorithms were paired, this paper used a paired-sample t-test. Of note, this paper did not care about the differences between other algorithms. Thus, it was essentially a pairwise comparison between both groups (EEGG vs. CSP+LDA, EEGG vs. CSP+Bayes, EEGG vs. CSP+SVM).

This paper performed three statistical analyses. Firstly, this paper compared the AUC and variance of the AUC of EEGG with other algorithms using all subjects' testing results (see Fig.3). Secondly, the subjects were partitioned into the group (a) and group (b) based on whether EEGG outperformed others (see Fig.4). This paper compared the AUC of EEGG with other algorithms within the group (a) 
and group (b) (see Fig.5). Thirdly, this paper compared the AUC of each algorithm between both groups (see Fig.6).

\section{Results}

\section{These results were annotated according to the experimental architecture.}

\subsection{Classification Performance}

(a) This paper compared the classification performance of EEGG with other classic algorithms and further explored its advantages, disadvantages, and theoretical reasons for the performance.

The purpose of this part of the experiments was to explore classification performance. Fig. 3a showed that EEGG significantly outperformed CSP+LDA and CSP+Bayes and might outperform $\mathrm{CSP}+\mathrm{SVM}$ using all subjects' results. [AUC, Paired-samples t-test: $0.834 \pm 0.079>0.800 \pm 0.111$, $\mathrm{p}<0.05 ; 0.834 \pm 0.079>0.662 \pm 0.159, \mathrm{p}<0.001 ; 0.834 \pm$ $0.079>0.806 \pm 0.109, p=0.057]$ What stands out in Fig. $3 \mathrm{a}$ was that EEGG's AUC appeared to be more concentrated. Therefore, this paper compared the variance of AUC among algorithms. This is a rather interesting outcome (see Fig.3b). The variance of AUC of EEGG is smaller than others, which means that EEGG maybe has better robustness.

Further analysis was necessary for EEGG's advantages, disadvantages. This paper divided the subjects into both groups based on whether EEGG outperformed others (see Fig.4). To determine whether the grouping is reasonable, further analysis of the data within the group revealed that EEGG significantly outperformed others in EEGG's advantaged group (a), "CSP+" significantly outperformed EEGG others in EEGG's advantaged group (b) except for CSP+Bayes. [Group (a), AUC, Paired-samples t-test: $0.825 \pm$ $0.074>0.745 \pm 0.094, \mathrm{p}<0.001 ; 0.825 \pm 0.074>0.591 \pm 0.104$ $\mathrm{p}<0.001 ; 0.825 \pm 0.074>0.750 \pm 0.091, \mathrm{p}<0.001$. Group (b), AUC, Paired-samples t-test: $0.847 \pm 0.087<0.873 \pm 0.090$, $\mathrm{p}<0.01 ; 0.847 \pm 0.087>0.760 \pm 0.173, \mathrm{p}=0.061 ; 0.847 \pm$ $0.087<0.881 \pm 0.087, \mathrm{p}<0.01$.]

If we now turn to Fig. 6, the subjects' data of group (b) were easier to classify correctly (see Fig. 6). There were only two reasons determining classification performance: the algorithm and the data itself. Therefore, this means group (b) data has more distinct features for classification and group (a) data contains more noise based on the same algorithm. Further, there were significant differences in "CSP+" between group (a) and group (b) (see Fig. 6). However, EEGG results showed no significant differences between groups (see Fig. 6). This suggests one interesting thing. "CSP+" algorithms in this paper were more sensitive to EEG data quality. They exhibited better classifier performance
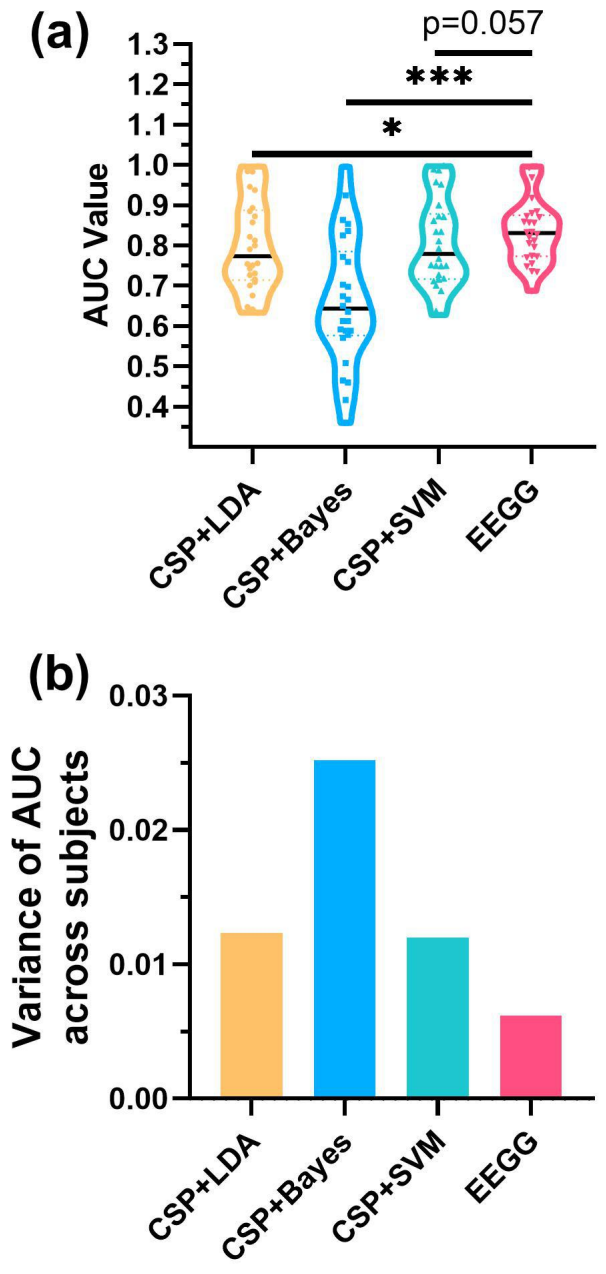

Figure 3. Comparison between EEGG and other algorithms using all testing results. (a) Comparison in AUC. (b) Comparison in variance of the AUC. ${ }^{*} p<0.05 .{ }^{* * *} p<0.001$.

than EEGG for data of better quality, and they performed poorly than EEGG for data of poor quality. In other words, EEGG was not sensitive to EEG data quality than "CSP+" algorithms. Although EEGG did not exhibit the highest classification accuracy, it performed better than others for data of poor quality (see Fig. 6). [AUC, Paired-samples t-test: CSP+LDA:0.745 $\pm 0.094<0.873 \pm 0.090, \mathrm{p}<0.01$; CSP+Bayes: $0.591 \pm 0.104<0.760 \pm 0.173, \mathrm{p}<0.01 ; \mathrm{CSP}+\mathrm{SVM}: 0.750 \pm$ $0.091<0.881 \pm 0.087, p<0.01 ;$ EEGG: $0.825 \pm 0.074<0.847 \pm$ $0.087, \mathrm{p}=0.499$ ]

Together, these above statistical analyses provided important insights that EEGG has better robustness for EEG data of poor quality (see Fig.4, Fig.5, and Fig.6). 

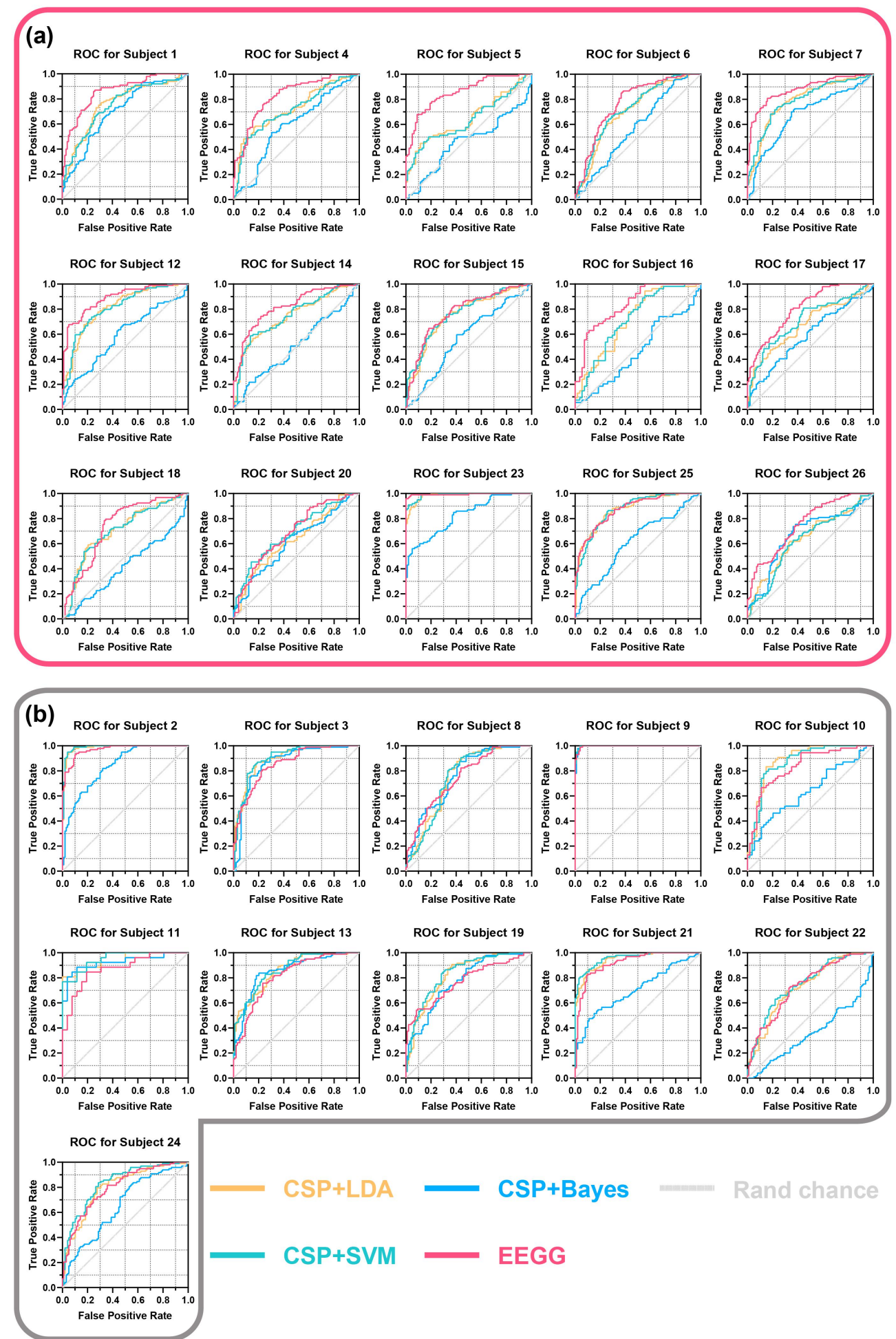

CSP+SVM $\longrightarrow$ EEGG

Figure 4. Partition based on whether EEGG outperformed others. (a) EEGG's advantaged group. (b) EEGG's disadvantaged group. These above figures were ROC curves. 

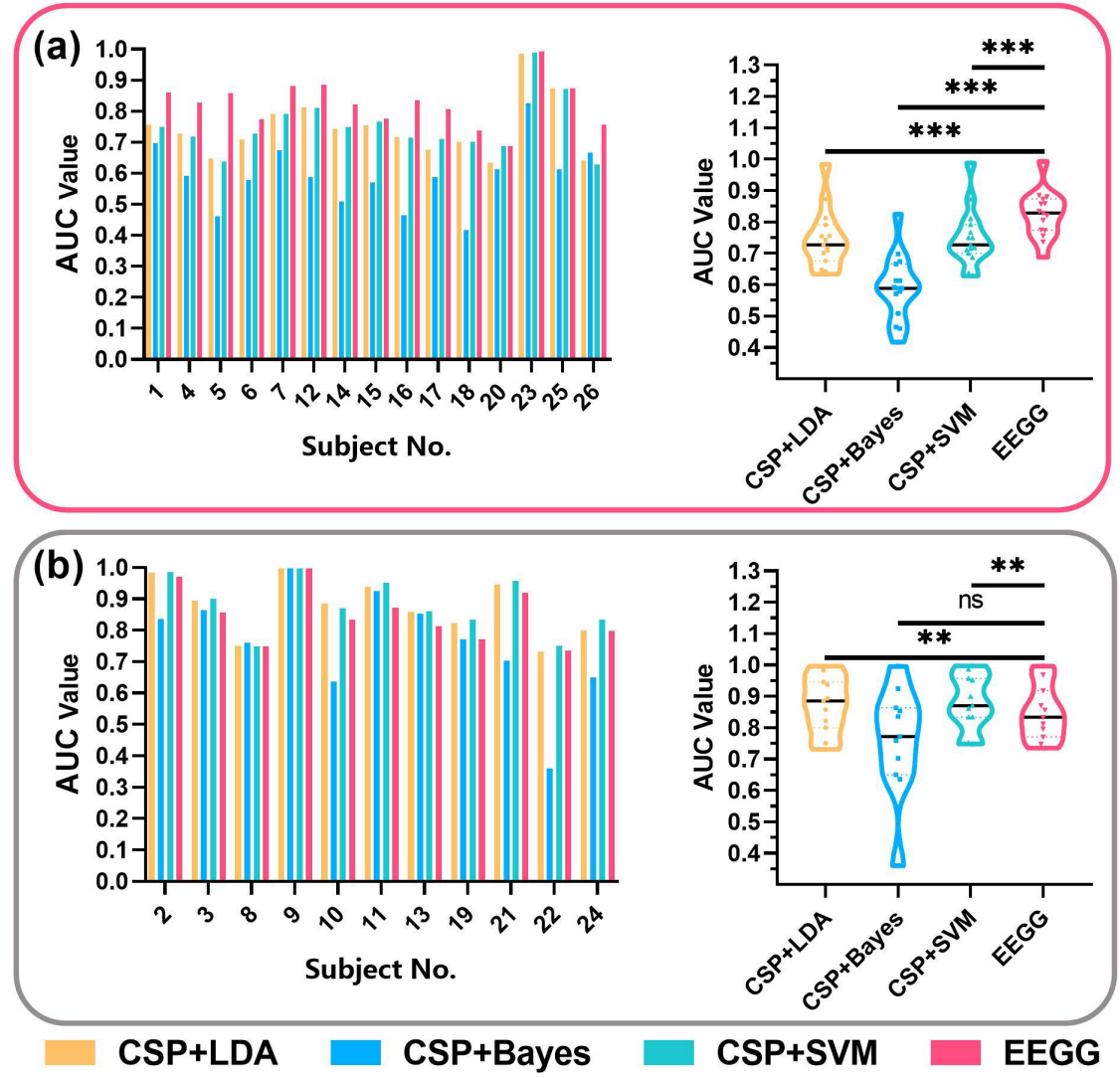

Figure 5. Comparison between EEGG and other algorithms within groups. (a) Comparison within group (a). (b) Comparison within group (b). ${ }^{* *} p<0.01 .{ }^{* * *} p<0.001 . n s>0.05$.

\subsection{Transformation of EEGG model and analysis of relation spectrum}

This section is divided into three parts: (1) transformed EEGG models and verification of the correctness of items, (2) statistical analysis of relation items in EEGG models and verification of the correctness using the known ERD/ERS phenomenon, and (3) the further BCI-based analysis of brain using relation spectrum.

Fig. 7 showed the EEGG models transformed into the relation spectrum without odd items. The abscissa $(P)$ of Fig. 7a is $E^{2}$, where $E$ was EEG signal. To verify the correctness of items, this paper calculated the total number of pairwise interaction, independent powers, and the squared of independent powers items $\left(C_{64}^{2}+64+64=2144\right)$ and calculated the correlation matrix between subjects' EEGG models (see Fig. 7b). The total number was equal to the number of items in the new relation spectrum form. The relation spectrums were similar across EEGG models of different subjects (see Fig. 7).

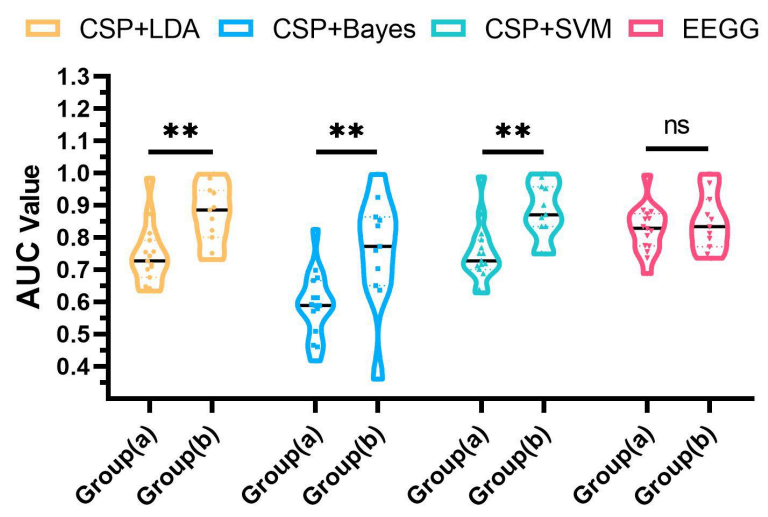

Figure 6. Comparison between groups. ${ }^{* *} p<0.01 . n s>0.05$.

(b) Since this paper decomposed the EEG intention system into a relation spectrum (Taylor-like series) for the first time, the rationality of EEGG decomposition was verified by exploring whether the components of the decomposed EEG-intention system contained the known ERD/ERS phenomenon. 
(a)

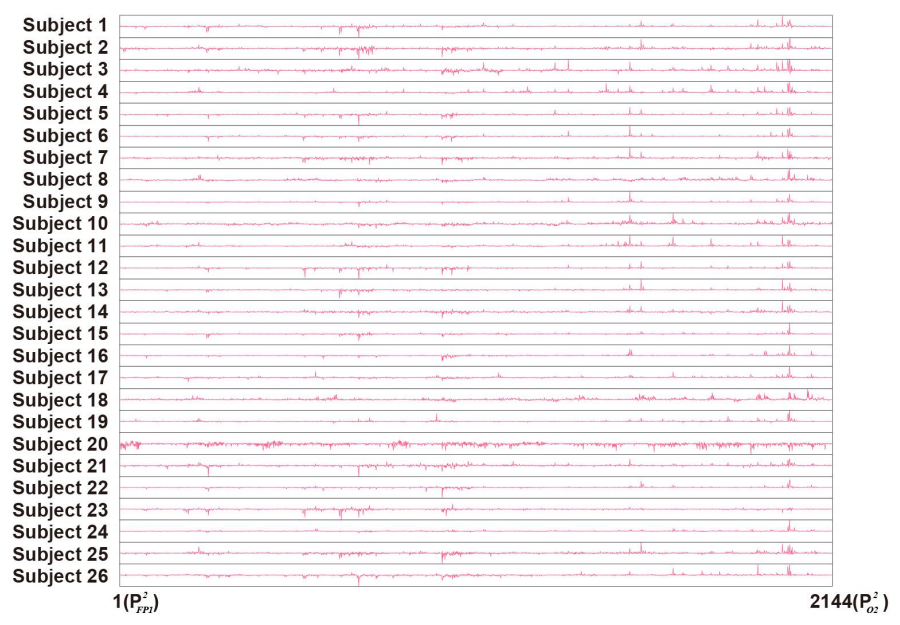

(b)

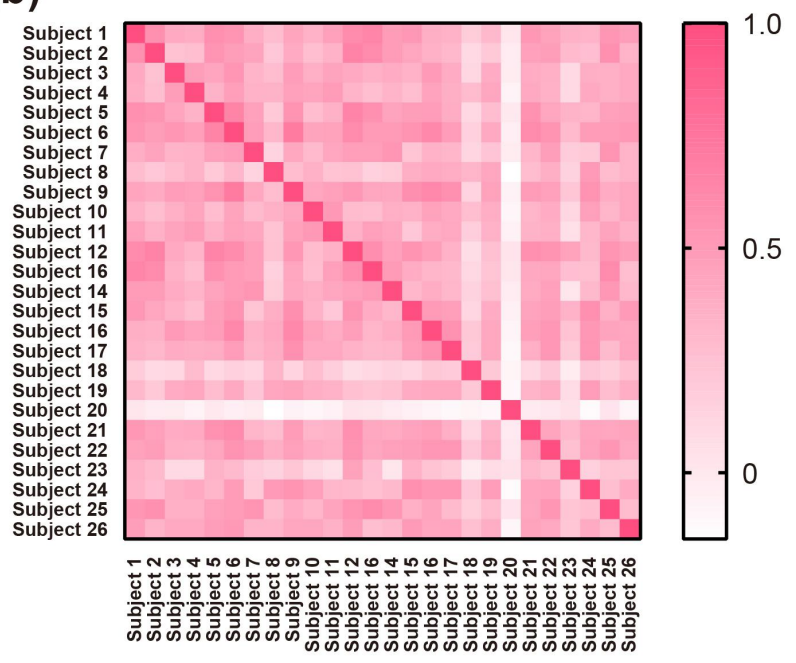

Figure 7. Transformed EEGG models. (a) The relation spectrum without odd items. (b) Correlation matrix between subjects' EEGG models. $P=E^{2}$, where $E$ is EEG signal.

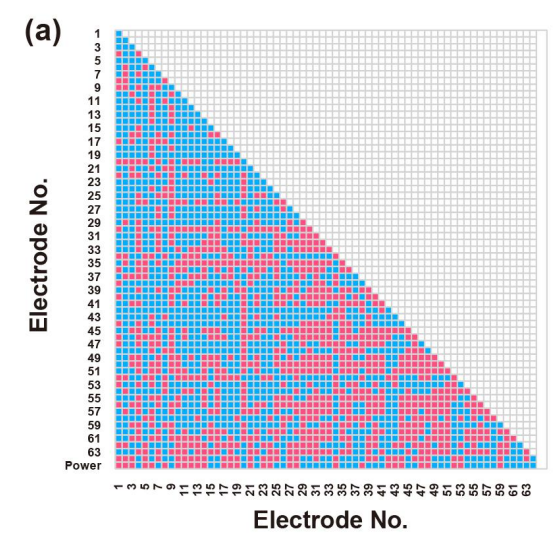

(b)

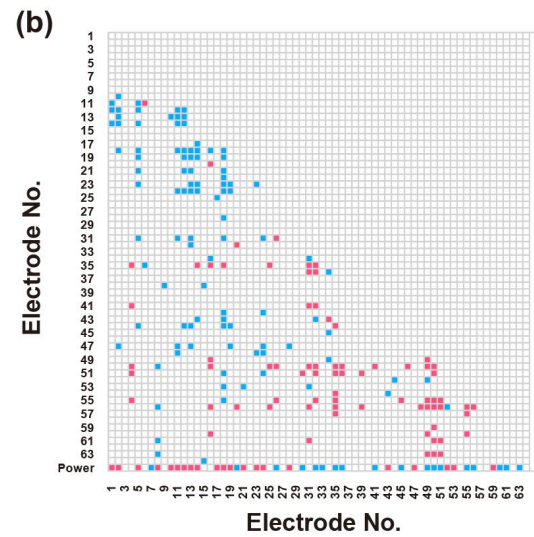

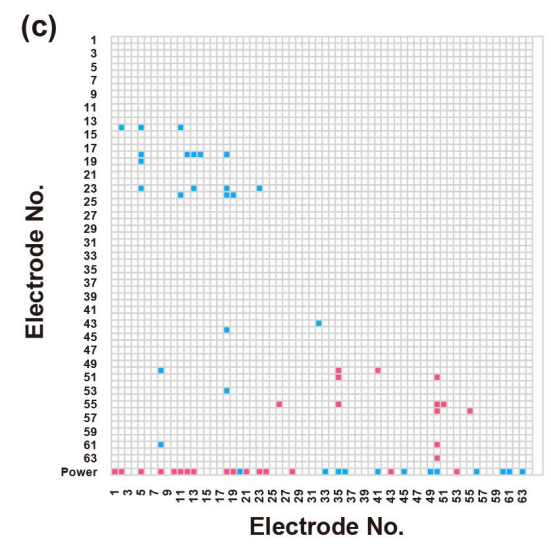

Figure 8. Results of statistical analysis of relation items in EEGG models. (b) Mean of relation items in EEGG models. (b) Results of statistical analysis under $p<0.01$. (c) Results of statistical analysis under $p<0.001$. "+": positive effect. "-": Negative effect. The upper triangular matrixes of the above figures were not used.

Turning now to the statistical analysis of relation items in EEGG models, we found some significant regularities, such as the symmetry of relation items (see Fig. 8). In order to make the regularities more intuitional, this paper visualized the EEGG model using the BrainNet Viewer (see Fig. 9). "Ball" expresses the independent components of brain regions, and "rod" expresses the interactive components of brain regions. The effects of "red ball," "blue ball," "blue rod," and "red rod" in Fig. 9a can be expressed as follows, respectively.

$$
\left\{\begin{array}{cc}
y^{\text {redball-effect }}=k_{\text {redball }} P_{\text {redball }} & \text { (see Fig.9b) } \\
y^{\text {blueball-effect }}=-k_{\text {blueball }} P_{\text {blueball }} & \text { (see Fig.9c) } \\
y^{\text {bluerod-effect }}=-k_{\text {bluerod }} P_{1 \text { bluerod }} P_{2 b l u e r o d} & \text { (see Fig.9d) } \\
y^{\text {redrod-effect }}=k_{\text {redrod }} P_{1 \text { re rod }} P_{2 \text { redrod }} & \text { (see Fig.9e) }
\end{array}\right.
$$

Where $y^{\text {redball-effect }}, y^{\text {blueball-effect }}, y^{\text {bluerod-effect }}$, and $y^{\text {redrod-effect }}$ expresses the effects of "red ball," "blue ball," "blue rod," and "red rod," respectively. $k_{\text {redball }} \in \mathbb{R}^{+}, k_{\text {blueball }} \in \mathbb{R}^{+}, k_{\text {bluerod }} \in \mathbb{R}^{+}$, and $k_{\text {redrod }} \in \mathbb{R}^{+}$are 


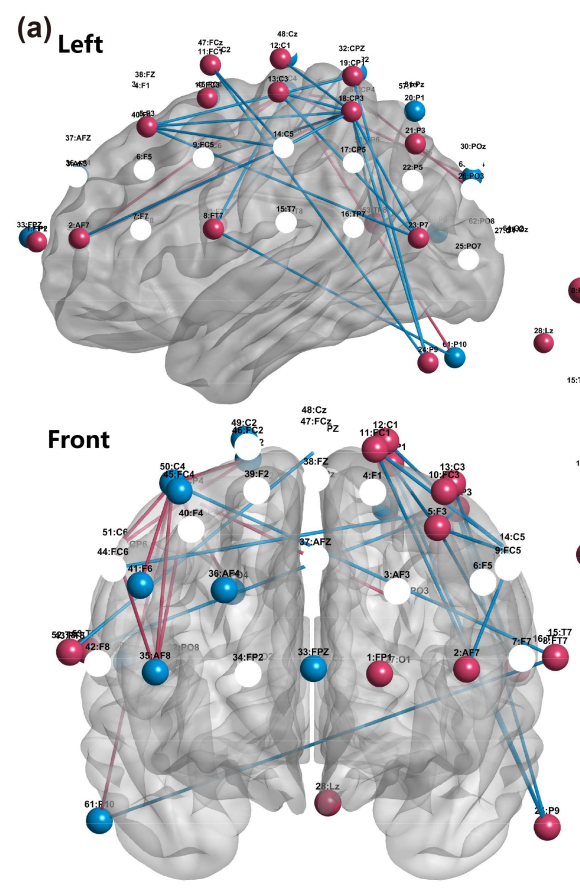

(b)

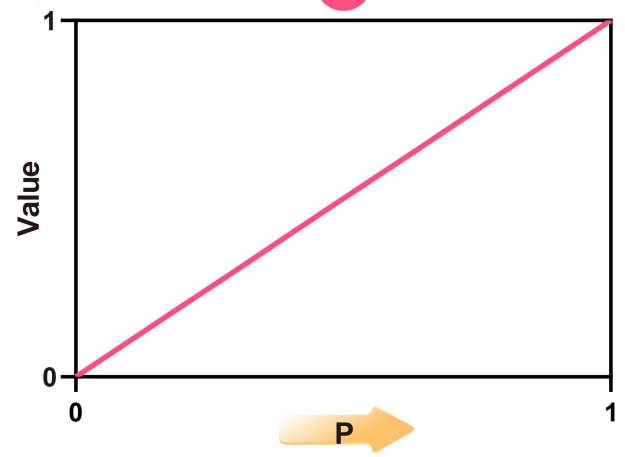

(d)

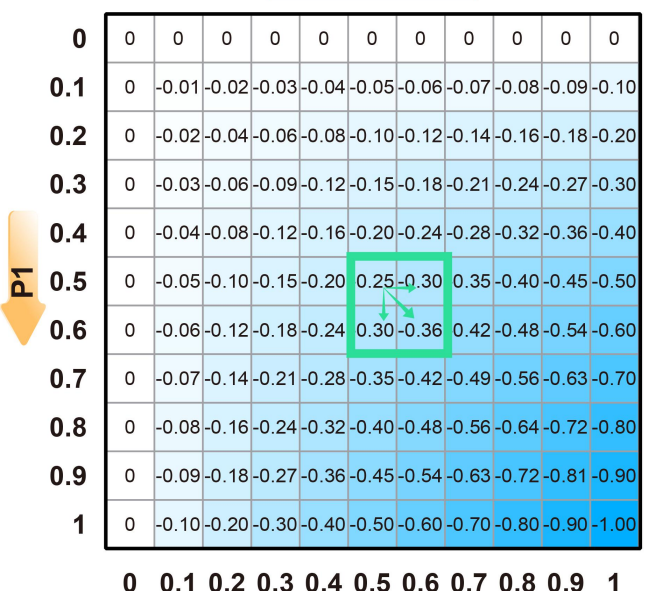

P2
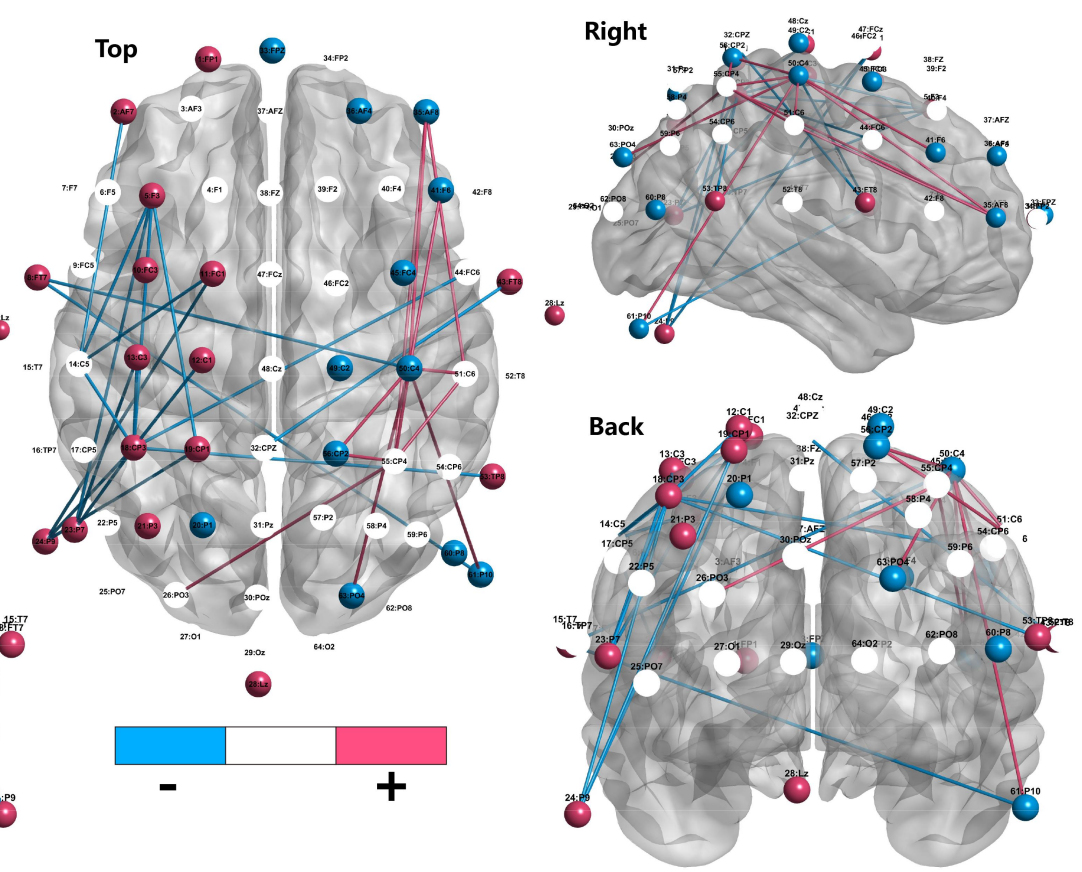

(c)

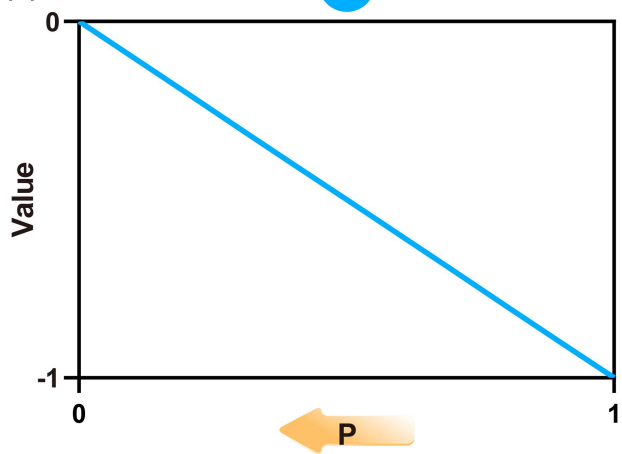

(e)
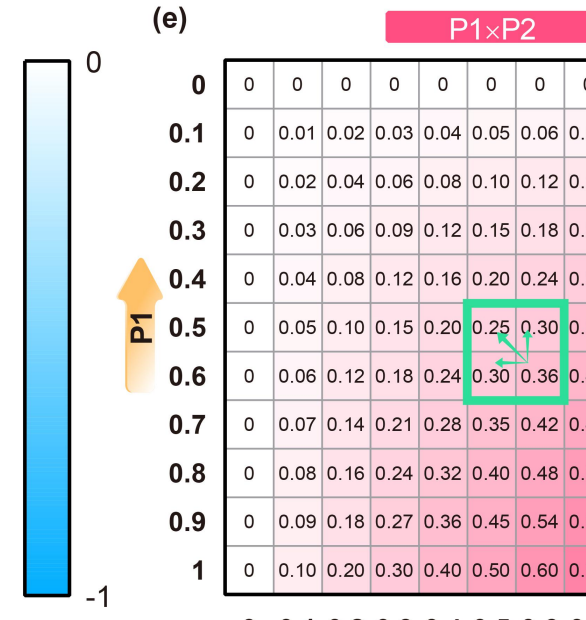

Figure 9. Visualization of the EEGG model under $p<0.001$. (a) The EEGG model. "Ball" expresses the independent components of brain regions, and "rod" expresses the interactive components of brain regions. (b) Graph of the effects of "red ball" in (a). (c) Graph of the effects of "blue ball" in (a). (d) Graph of the effects of "blue rod" in (a). (e) Graph of the effects of "red rod" in (a). Fig. 9b, c, d, e were the graph of $E q .(7)$ for describing the the effects of relation items. 
proportional coefficients. $P_{\text {redball }} \in[0,1], \quad P_{\text {blueball }} \in[0,1]$, $P_{1 \text { bluerod }} \in[0,1], \quad P_{2 \text { bluerod }} \in[0,1], \quad P_{1 \text { redrod }} \in[0,1], \quad$ and $P_{\text {redrod }} \in[0,1]$ represents powers. $P_{\text {redball }}$ and $P_{\text {blueball }}$ express the independent components. $P_{1 b l u e r o d} P_{2 b l u e r o d}$ and $P_{1 \text { redrod }} P_{2 \text { redrod }}$ express the interactive components. To show the change of the above effects, this paper plotted the change when proportional coefficients were 1 (see Fig. 9b, Fig. 9c, Fig. 9d, and Fig. 9e). Next, this paper verified the known ERD/ERS phenomenon and showed a new phenomenon [34].

The positive output of EEGG model in this paper represented motor imagery of the left hand. In the subsequent analysis, the motor imagery of the left hand was shown as examples. We assume that subject imagines the left-hand movement, the power of the right hemisphere will decrease (ERD), and the power of the left hemisphere will increase (ERS) according to the known ERD/ERS phenomenon [34]. If the relation spectrum was reasonable, the EEGG model's output would tend to be positive (That is, this brain state tended to be classified as left-handed motor imagery by the EEGG model or relation spectrum.) when the above phenomenon occurs.

It happened that most "blue balls" distribute on the right hemisphere, and most "red balls" distribute on the left hemisphere (see Fig. 9a). The EEGG model's output increases with decreasing power of "blue ball" (see yellow arrow in Fig. 9c), which is the ERD. Likewise, the EEGG model's output increases with increasing power of "red ball" (see yellow arrow in Fig. 9b), which is the ERS.

(c) Based on rationality, this paper explored the previously unreachable further BCI-based analysis of brain.

Additionally, it happened that most "red rods" distribute on the right hemisphere, and most "blue rods" distribute on the left hemisphere (see Fig. 9a). The effects of "rods" put a brake on the effects of "balls." In Fig. 9e, we found the EEGG model's output decreases with decreasing power in both ends of "red rod" (see yellow arrow in Fig. 9e). In Fig. $9 \mathrm{~d}$, we found the EEGG model's output decreases with increasing power in both ends of "blue rod" (see yellow arrow in Fig. 9d). In order to slow the rate of decline, the power of both connected "balls" should not change at the same time as possible, because the most gradient was on the diagonal line (see Fig. 9d and Fig. 9e). This implies that the activation in the brain should be more centralized to be identified as the motor imagery of the left hand.

\section{Discussion}

The human brain is a complex interconnected dynamical system [41]. Since the first BCI has been proposed, efforts were then paid to decipher the human brain. This paper presents the analytic "white box" Brain-computer interface algorithm named EEGG that decomposed the EEG-intention system into relation spectrum (Taylor-like series) for the first time, which offers a novel frame for analysis of the brain. Through experiments, this paper explored the classification performance of EEGG, verified the rationality of the relation spectrum, and then tried to analyze further the brain based on the relation spectrum.

The following theoretical analysis explained the results.

\subsection{The controllable logical expression capacity of EEGG makes it better robust for EEG data of poor quality.}

EEG is the most common brain signal that has been utilized in BCI applications because of its non-invasive nature and low cost $[42,43]$. However, it is well known that EEG has a weak signal-to-noise ratio due to the acquisition points on the scalp [25, 42, 43]. Most ML algorithms aim to get more excellent expression ability [19]. Nevertheless, it is easy to cause over-fitting for the EEG data of poor quality using ML algorithms with too excellent expression ability. EEG-ML algorithms' expression ability should happen to meet the expression of a small amount of classified information and do not have the ability to express a large amount of other information. That is, the ML algorithm with outstanding approximation or expression ability may not be suitable for the EEG data of poor quality, which may also be the reason why deep learning methods have not yet shown convincing improvement [3].

Dendrite Net presented by Gang Liu et al. in 2020 has controllable logical expression capacity, which happens to meet the needs of the character of EEG with a low signal-tonoise ratio [23]. Dendrite Net aims to design the logical extractor with controllable logical expression capacity. Concretely, its logical expression capacity can be adjusted by the number of dendrite modules. In this paper, we used the enhanced DD, ResDD of Gang neuron [24]. ResDD is an improved Dendrite Net using a residual strategy to prevent a vanishing gradient. The logical expression capacity of ResDD modules is the same as the DD module. In the experiments, this paper only uses one ResDD module; thus, after taking the power calculation of EEGG into account, the highest order of the EEGG model in this paper is 4 (see Fig. 1 and Fig. 2). The constant logical expression capacity made the EEGG model avoid the overfitting phenomenon and improved the robustness for EEG data of poor quality (see Fig. 6). Meanwhile, the corresponding disadvantage is that the constant logical expression capacity cannot express some details and made EEGG not exhibit the highest classification accuracy for EEG data of better quality (see Fig. 6). Fortunately, the logical expression capacity of EEGG is controllable. Thus, researchers can adjust it based on the actual conditions. 


\subsection{The EEG-intention system was decomposed into Taylor-like series.}

EEGG model consists of Spatial filter, Power calculation, and ResDD Neural Network. In the Transformation of EEGG model, the power of EEG was calculated by the magnitude squared instead of the variance or standard error. Inheriting ResDD of Gang neuron [24, 44], EEGG implemented nonlinear operations by the Matrix product and Hadamard product. Therefore, the relation spectrum can be obtained through formula simplification of Eq.(6) in software (e.g., MATLAB or Python). This paper found the known $\mathrm{ERD} / \mathrm{ERS}$ phenomenon in relation spectrum and verified the correctness of the transformed EEGG model(see Fig. 9). Further, because the EEGG model's results were obtained under $\mathrm{t}$-test with $\mathrm{p}<0.001$, this paper drew the activation in the brain should be more centralized for fine hand intention from Fig. 9. In other words, fine motion corresponded to a centralized region. This finding was similar to the anatomy study [45].

\section{3 "Decomposition" in this paper and interpretation are easily-confused.}

"Interpretation" in the existing BCI algorithms refers to explain the outputs of the black-box model (e.g., deep neural networks) [17]. "Decomposition" refers to decompose an unknown composition into some simple components with physical meaning (e.g., Fourier transform and Fourier spectrum for decomposing signals) and read parameters of the white-box model, that is, simple components [22].

The interpretability of ML outputs has been studied extensively in computer vision and natural language processing (e.g., feature visualization for deep networks [12, 13], attribution for convolutional networks [14, 15], and dimensionality reduction [16]). Interpretable approaches are used to explain the BCI model's outputs in recent years [1720]. For instance, Layerwise relevance propagation (LRP) was used to explain individual deep network decisions/outputs in the literature [17]. Additionally, most linear algorithms are interpretable. However, few EEG-BCI models use only a linear algorithm, except for the data with fewer channels or simpler logic relationships [20]. After all, the relationship between EEG signals and intention is nonlinear, and the general $\mathrm{BCI}$ algorithm is composed of a linear algorithm and a nonlinear classifier.

This paper emphasized the decomposition of the biological EEG-intention system. It is widely known that a functional relationship can be expressed by the sum of trigonometric or power polynomial. For the expression of a trigonometric polynomial, the typical example is Fourier frequency spectrum. Here, the relation spectrum is the spectrum of the power series. EEGG used in the analysis can be similar to Fourier transform. However, Fourier Transform was used for signal decomposition, while EEGG was used to decompose the EEG-intention system.

There are also limitations to this paper. This paper did not contrast deep learning methods. Nevertheless, this paper did not emphasize that the classification accuracy was better than SOAT because this paper paid more attention to the specific characters of theoretical proof (the controllable logical expression capacity for classification). Besides, it mainly emphasized EEGG's analysis capacity. This paper is the first decomposition with Taylor-like series on the EEG-intention system. EEGG can decompose the brain intentions (system), just like the Fourier transform that decomposes signal. Additionally, in order to verify the correctness of transformed EEGG models, this paper selected the MI paradigms with the known ERD/ERS phenomenon. Multiclass classification of EEGG will be explored in future research. Because the transformed EEGG model's correctness has been verified and the Transformation was the same for both and multiclass classification, the multiclass EEGG models can be analyzed in future research.

\section{Conclusion}

A "white box" brain-computer interface algorithm named EEGG that decomposed the EEG-intention system into relation spectrum (Taylor-like series) for the first time was proposed in the paper, which offers a novel frame for analysis of the brain. EEGG can be used for intention recognition and analysis of the composition of biological EEG-intention system. The conclusions of major questions of the algorithm/frame proposed in the Introduction section are as follows.

(1) Experiments and theoretical analysis showed EEGG had better robustness for EEG data of poor quality.

(2) Experiments demonstrated the known ERD/ERS existed in relation spectrum.

(3) Based on the relation spectrum, this paper found that the activation in the brain should be more centralized for fine hand intention.

Before closing, it is worth mentioning that decomposing EEG-intention systems into Taylor-like series via EEGG may find some important findings of brain in the future.

\section{Acknowledgements}

Gang Liu presented EEGG. Jing Wang provided advice. Gang neuron using in this paper could be found at https://github.com/liugang1234567/Gang-neuron.

\section{References}

[1] M. A. L. Nicolelis, "Actions from thoughts," Nature, vol. 409, no. 6818, pp. 403-407, 2001/01/01, 2001. 
[2] L. F. Nicolas-Alonso, and J. Gomez-Gil, "Brain Computer Interfaces, a Review," Sensors, vol. 12, no. 2, pp. 1211-1279, Feb, 2012.

[3] F. Lotte, L. Bougrain, A. Cichocki, M. Clerc, M. Congedo, A. Rakotomamonjy, and F. Yger, "A review of classification algorithms for EEG-based brain-computer interfaces: a 10 year update," Journal of Neural Engineering, vol. 15, no. 3, pp. 28, Jun, 2018.

[4] O. Lo-Thong, P. Charton, X. F. Cadet, B. GrondinPerez, E. Saavedra, C. Damour, and F. Cadet, "Identification of flux checkpoints in a metabolic pathway through white-box, grey-box and blackbox modeling approaches," Scientific Reports, vol. 10, no. 1, Aug 10, 2020.

[5] X. W. Li, and J. Wen, "Review of building energy modeling for control and operation," Renewable \& Sustainable Energy Reviews, vol. 37, pp. 517-537, Sep, 2014.

[6] X. S. Wang, and O. Gotoh, "Accurate molecular classification of cancer using simple rules," Bmc Medical Genomics, vol. 2, pp. 23, Oct, 2009.

[7] R. Sitaram, H. H. Zhang, C. T. Guan, M. Thulasidas, Y. Hoshi, A. Ishikawa, K. Shimizu, and N. Birbaumer, "Temporal classification of multichannel near-infrared spectroscopy signals of motor imagery for developing a brain-computer interface," Neuroimage, vol. 34, no. 4, pp. 14161427, Feb, 2007.

[8] Y. Jiao, T. Zhou, L. Yao, G. Zhou, X. Wang, and Y. Zhang, "Multi-view Multi-scale Optimization of Feature Representation for EEG Classification Improvement," IEEE transactions on neural systems and rehabilitation engineering : $a$ publication of the IEEE Engineering in Medicine and Biology Society, vol. PP, 2020-Nov-27, 2020.

[9] C. C. Chang, and C. J. Lin, "LIBSVM: A Library for Support Vector Machines," Acm Transactions on Intelligent Systems and Technology, vol. 2, no. 3, pp. 27, 2011.

[10] Q. Wang, G. M. Garrity, J. M. Tiedje, and J. R. Cole, "Naive Bayesian classifier for rapid assignment of rRNA sequences into the new bacterial taxonomy," Applied and Environmental Microbiology, vol. 73, no. 16, pp. 5261-5267, Aug, 2007.

[11] C. Zhang, and A. Eskandarian, "A Computationally Efficient Multiclass Time-Frequency Common Spatial Pattern Analysis on EEG Motor Imagery," Annual International Conference of the IEEE Engineering in Medicine and Biology Society. IEEE Engineering in Medicine and Biology Society. Annual International Conference, vol. 2020, pp. 514-518, 2020-07, 2020.

[12] D. Erhan, Y. Bengio, A. Courville, and P. Vincent, "Visualizing higher-layer features of a deep network," University of Montreal, vol. 1341, no. 3, pp. 1, 2009.
[13] A. Nguyen, J. Yosinski, J. Clune, and Ieee, "Deep Neural Networks are Easily Fooled: High Confidence Predictions for Unrecognizable Images," 2015 Ieee Conference on Computer Vision and Pattern Recognition, IEEE Conference on Computer Vision and Pattern Recognition, pp. 427436, New York: Ieee, 2015.

[14] M. D. Zeiler, and R. Fergus, "Visualizing and understanding convolutional networks." pp. 818833.

[15] J. T. Springenberg, A. Dosovitskiy, T. Brox, and M. Riedmiller, "Striving for simplicity: The all convolutional net," arXiv preprint arXiv:1412.6806, 2014.

[16] L. Van der Maaten, and G. Hinton, "Visualizing data using t-SNE," Journal of machine learning research, vol. 9, no. 11, 2008.

[17] I. Sturm, S. Lapuschkin, W. Samek, and K. R. Muller, "Interpretable deep neural networks for single-trial EEG classification," Journal of Neuroscience Methods, vol. 274, pp. 141-145, Dec, 2016.

[18] A. Farahat, C. Reichert, C. M. Sweeney-Reed, and H. Hinrichs, "Convolutional neural networks for decoding of covert attention focus and saliency maps for EEG feature visualization," Journal of Neural Engineering, vol. 16, no. 6, pp. 14, Dec, 2019.

[19] R. T. Schirrmeister, J. T. Springenberg, L. D. J. Fiederer, M. Glasstetter, K. Eggensperger, M. Tangermann, F. Hutter, W. Burgard, and T. Ball, "Deep learning with convolutional neural networks for EEG decoding and visualization," Human brain mapping, vol. 38, no. 11, pp. 5391-5420, 2017.

[20] S. Haufe, F. Meinecke, K. Görgen, S. Dähne, J.-D. Haynes, B. Blankertz, and F. Bießmann, "On the interpretation of weight vectors of linear models in multivariate neuroimaging," Neuroimage, vol. 87, pp. 96-110, 2014.

[21] M. R. Joglekar, L. Chariker, R. Shapley, and L. S. Young, "A case study in the functional consequences of scaling the sizes of realistic cortical models," Plos Computational Biology, vol. 15, no. 7, pp. 25, Jul, 2019.

[22] P. D. Welch, "USE OF FAST FOURIER TRANSFORM FOR ESTIMATION OF POWER SPECTRA - A METHOD BASED ON TIME AVERAGING OVER SHORT MODIFIED PERIODOGRAMS," Ieee Transactions on Audio and Electroacoustics, vol. AU15, no. 2, pp. 70-+, 1967, 1967.

[23] G. Liu, and J. Wang, "Dendrite Net: A White-Box Module for Classification, Regression, and System Identification," arXiv e-prints, https://arxiv.org/abs/2004.03955, [April 01, 2020, 2020]. 
[24] L. Gang, "It may be time to improve the neuron of artificial neural network," TechRxiv. Preprint., https://doi.org/10.36227/techrxiv.12477266, 2020].

[25] B. Blankertz, R. Tomioka, S. Lemm, M. Kawanabe, and K. R. Muller, "Optimizing spatial filters for robust EEG single-trial analysis," Ieee Signal Processing Magazine, vol. 25, no. 1, pp. 41-56, Jan, 2008.

[26] P. L. Nunez, R. Srinivasan, A. F. Westdorp, R. S. Wijesinghe, D. M. Tucker, R. B. Silberstein, and P. J. Cadusch, "EEG coherency: I: statistics, reference electrode, volume conduction, Laplacians, cortical imaging, and interpretation at multiple scales," Electroencephalography and Clinical Neurophysiology, vol. 103, no. 5, pp. 499-515, 1997/11/01/, 1997.

[27] A. Kachenoura, L. Albera, L. Senhadji, and P. Comon, "ICA: A potential tool for BCI systems," Ieee Signal Processing Magazine, vol. 25, no. 1, pp. 57-68, Jan, 2008.

[28] G. Pfurtscheller, C. Neuper, D. Flotzinger, and M. Pregenzer, "EEG-based discrimination between imagination of right and left hand movement," Electroencephalography and clinical Neurophysiology, vol. 103, no. 6, pp. 642-651, 1997.

[29] A. Gidon, T. A. Zolnik, P. Fidzinski, F. Bolduan, A. Papoutsi, P. Poirazi, M. Holtkamp, I. Vida, and M. E. Larkum, "Dendritic action potentials and computation in human layer $2 / 3$ cortical neurons," Science, vol. 367, no. 6473, pp. 83-+, Jan, 2020.

[30] K. He, X. Zhang, S. Ren, and J. Sun, "Deep Residual Learning for Image Recognition." pp. 770778.

[31] M. R. Xia, J. H. Wang, and Y. He, "BrainNet Viewer: A Network Visualization Tool for Human Brain Connectomics," Plos One, vol. 8, no. 7, pp. 15, Jul, 2013.

[32] H. Cho, M. Ahn, S. Ahn, M. Kwon, and S. C. Jun, "EEG datasets for motor imagery brain-computer interface," Gigascience, vol. 6, no. 7, pp. 1-8, May, 2017.

[33] K. Wang, M. P. Xu, Y. J. Wang, S. S. Zhang, L. Chen, and D. Ming, "Enhance decoding of premovement EEG patterns for brain-computer interfaces," Journal of Neural Engineering, vol. 17, no. 1, pp. 11, Feb, 2020.

[34] G. Pfurtscheller, and F. H. L. da Silva, "Eventrelated EEG/MEG synchronization and desynchronization: basic principles," Clinical Neurophysiology, vol. 110, no. 11, pp. 1842-1857, Nov, 1999.

[35] X. K. Shu, S. G. Chen, L. Yao, X. J. Sheng, D. G. Zhang, N. Jiang, J. Jia, and X. Y. Zhu, "Fast Recognition of BCI-Inefficient Users Using Physiological Features from EEG Signals: A Screening Study of Stroke Patients," Frontiers in Neuroscience, vol. 12, pp. 12, Feb, 2018.
[36] R. Zhang, D. Yao, P. A. Valdes-Sosa, F. Li, P. Li, T. Zhang, T. Ma, Y. Li, and P. Xu, "Efficient restingstate EEG network facilitates motor imagery performance," Journal of Neural Engineering, vol. 12, no. 6, Dec, 2015.

[37] C. Neuper, R. Scherer, M. Reiner, and G. Pfurtscheller, "Imagery of motor actions: Differential effects of kinesthetic and visual-motor mode of imagery in single-trial EEG," Cognitive Brain Research, vol. 25, no. 3, pp. 668-677, Dec, 2005.

[38] H. Cho, M. Ahn, and S. Ahn, "Supporting data for "EEG datasets for motor imagery brain computer interface."," GigaScience Database, 2017.

[39] X. Zhao, J. Zhao, C. Liu, and W. Cai, "Deep Neural Network with Joint Distribution Matching for Cross-Subject Motor Imagery Brain-Computer Interfaces," Biomed Research International, vol. 2020, Feb 24, 2020.

[40] V. Martínez-Cagigal. "Common Spatial Patterns (CSP),"

https://www.mathworks.com/matlabcentral/fileexch ange/72204-common-spatial-patterns-csp.

[41] Y. He, A. Dagher, Z. Chen, A. Charil, A. Zijdenbos, K. Worsley, and A. Evans, "Impaired small-world efficiency in structural cortical networks in multiple sclerosis associated with white matter lesion load," Brain, vol. 132, pp. 3366-3379, Dec, 2009.

[42] F. Lotte, M. Congedo, A. Lecuyer, F. Lamarche, and B. Arnaldi, "A review of classification algorithms for EEG-based brain-computer interfaces," Journal of Neural Engineering, vol. 4, no. 2, pp. R1-R13, Jun, 2007.

[43] U. Chaudhary, N. Birbaumer, and A. RamosMurguialday, "Brain-computer interfaces for communication and rehabilitation," Nature Reviews Neurology, vol. 12, no. 9, pp. 513-525, Sep, 2016.

[44] G. Liu, and J. Wang, "A Relation Spectrum Inheriting Taylor Series: Muscle Synergy and Coupling for Hand," arXiv e-prints, https://ui.adsabs.harvard.edu/abs/2020arXiv200411 910L, [April 01, 2020, 2020].

[45] M. S. A. Graziano, "Etholocical Action Maps: A Paradigm Shift for the Motor Cortex," Trends in Cognitive Sciences, vol. 20, no. 2, pp. 121-132, Feb, 2016. 